\title{
Early whole-brain CT perfusion for detection of patients at risk for delayed cerebral ischemia after subarachnoid hemorrhage
}

\author{
Vesna Malinova, MD, ${ }^{1}$ Karoline Dolatowski, MD, ${ }^{2}$ Peter Schramm, MD, ${ }^{2,4}$ Onnen Moerer, MD, ${ }^{3}$ \\ Veit Rohde, MD, ${ }^{1}$ and Dorothee Mielke, MD' ${ }^{1}$
}

Departments of ${ }^{1}$ Neurosurgery, ${ }^{2}$ Neuroradiology, and ${ }^{3}$ Anaesthesiology, Georg-August-University, Göttingen; and ${ }^{4}$ Department of Neuroradiology, University of Lübeck, Germany

\begin{abstract}
OBJECTIVE This prospective study investigated the role of whole-brain CT perfusion (CTP) studies in the identification of patients at risk for delayed ischemic neurological deficits (DIND) and of tissue at risk for delayed cerebral infarction (DCl).

METHODS Forty-three patients with aneurysmal subarachnoid hemorrhage (aSAH) were included in this study. A CTP study was routinely performed in the early phase (Day 3). The CTP study was repeated in cases of transcranial Doppler sonography (TCD)-measured blood flow velocity (BFV) increase of $>50 \mathrm{~cm} / \mathrm{sec}$ within 24 hours and/or on Day 7 in patients who were intubated/sedated.
\end{abstract}

RESULTS Early CTP studies revealed perfusion deficits in 14 patients, of whom 10 patients (72\%) developed DIND, and 6 of these 10 patients $(60 \%)$ had $\mathrm{DCl}$. Three of the 14 patients $(21 \%)$ with early perfusion deficits developed $\mathrm{DCl}$ without having had DIND, and the remaining patient $(7 \%)$ had neither DIND nor DCI. There was a statistically significant correlation between early perfusion deficits and occurrence of DIND and $\mathrm{DCl}(\mathrm{p}<0.0001)$. A repeated CTP was performed in 8 patients with a TCD-measured BFV increase $>50 \mathrm{~cm} / \mathrm{sec}$ within 24 hours, revealing a perfusion deficit in 3 of them (38\%). Two of the 3 patients (67\%) developed DCI without preceding DIND and 1 patient (33\%) had DIND without DCI. In 4 of the 7 patients (57\%) who were sedated and/or comatose, additional CTP studies on Day 7 showed perfusion deficits. All 4 patients developed DCl.

CONCLUSIONS Whole-brain CTP on Day 3 after aSAH allows early and reliable identification of patients at risk for DIND and tissue at risk for DCl. Additional CTP investigations, guided by TCD-measured BFV increase or persisting coma, do not contribute to information gain.

http://thejns.org/doi/abs/10.3171/2015.6.JNS15720

KEY WORDS subarachnoid hemorrhage; delayed ischemic neurological deficit; CT perfusion; imaging; vasospasm; transcranial Doppler sonography; vascular disorders

$\mathrm{C}$ EREBRAL infarction with permanent vasospasminduced delayed ischemic neurological deficits (DIND) after aneurysmal subarachnoid hemorrhage (aSAH) is a feared and unfortunately frequent complication, responsible for poor functional and overall outcome. ${ }^{2}$ The pathophysiology of DIND and vasospasminduced delayed cerebral infarction (DCI) after aSAH is multifactorial and still not fully understood. It is well accepted that vasospasm is a major contributor to DIND and
DCI, but it is also assumed that processes already initiated shortly after aSAH render some brain areas more vulnerable for vasospasm than others. ${ }^{20,27,33}$ In up to $15 \%$ of patients, cerebral infarction and a fixed neurological deficit (including death) are irreversible end points of a dynamic process starting with tissue predisposition and asymptomatic vasospasm, followed by symptomatic vasospasm and transient DIND. For the successful prevention of infarction by antivasospastic therapy, timely recognition of

ABBREVIATIONS aSAH = aneurysmal subarachnoid hemorrhage; $\mathrm{BFV}=$ blood flow velocity; $\mathrm{CBF}=$ cerebral blood flow; $\mathrm{CBV}=$ cerebral blood volume; $\mathrm{CTA}=\mathrm{CT}$ angiography; CTP = CT perfusion; $\mathrm{DCl}=$ delayed cerebral infarction; DIND = delayed ischemic neurological deficits; DSA = digital subtraction angiography; MIP = maximum-intensity projection; MTT = mean transit time; NPV = negative predictive value; PPV = positive predictive value; $R O I=$ region of interest; $T C D=$ transcranial Doppler sonography; TTP = time to peak; TTS = time to start.

SUBMITTED March 31, 2015. ACCEPTED June 18, 2015.

INCLUDE WHEN CITING Published online December 18, 2015; DOI: 10.3171/2015.6.JNS15720. 
tissue at risk is critical, but also challenging due to a multitude of factors. This can result in neurological decline because an examination is of limited value in patients with a poor Hunt and Hess grade, as well as in patients who are sedated and/or comatose.

Various diagnostic tools have been applied to detect patients with cerebral vasospasm after aSAH. ${ }^{3,12,17,18,21,28,29}$ The ideal diagnostic tool should be noninvasive, easy, fast, and repeatable, and should allow not only the identification of patients with vasospasm but also of patients at risk for DIND and DCI. Digital subtraction angiography (DSA) is considered the gold standard for the diagnosis of cerebral vasospasm, but its invasiveness precludes its use for monitoring purposes. Transcranial Doppler sonography (TCD) is a widely used, noninvasive bedside method that is suitable for daily monitoring. $5,8,11,18,22,27,30,32$ However, TCD is dependent on the experience of the investigator and does not allow the detection of peripheral vessel vasospasm. Without clinical information, DSA and TCD do not allow identification of the progression of asymptomatic to symptomatic vasospasm and identification of tissue at risk. ${ }^{18,30}$

Computed tomography perfusion (CTP) allows visualization of brain perfusion and has been applied in brain tumors, ischemic stroke, and aSAH. . $^{1,410,12,14,15,17-19,23,25,28,33,36-38}$ A disadvantage of CTP is the exposure to radiation, which precludes its frequent use. This is reflected by available studies, in which CTP was performed once between Days 5 and 14, which represent the days with the highest probability of vasospasm occurrence. 1,4,12,15,17,18,23,28,29,35,36 A correlation between hypoperfusion in CTP and vasospastic infarction was found. However, the retrospective design of these studies, with inappropriate imaging time points and perfusion measurements in preselected regions instead of global brain perfusion, failed to show a therapeutically relevant correlation between hypoperfusion and tissue at risk for stroke development. 4,12,15,17,18,23,29,35,36

Recently, whole-brain CTP became available, which might at least overcome the problem of focal perfusion measurement. ${ }^{10,25,37,38}$ The aim of this prospective study was to evaluate the role of whole-brain CTP for the identification of tissue at risk for DCI and, thereby, of patients at risk for developing DIND and/or vasospasm-associated infarction, either 1) early after aSAH, prior to the vasospasm period; 2) during rapidly progressive TCD-confirmed vasospasm with or without symptoms; and/or 3) on Day 7 in patients who were comatose or sedated and could not be neurologically assessed.

\section{Methods}

A total of 43 patients with aSAH, who were admitted between August 2012 and December 2013, were enrolled in this prospective single-center study. The local institutional review board approved the study protocol. Patients who were younger than 18 years, pregnant, had severe renal dysfunction, or admitted later than 24 hours after bleeding were excluded. All patients were treated according to the institute's standard protocol for management of patients with aSAH. All procedures followed were in accordance with the ethical standards of the responsible committee on human experimentation (institutional and national) and with the Helsinki Declaration of 1975, as revised in $2008 .^{34}$

The diagnosis of aSAH was confirmed by a CT scan and CT angiography (CTA) and/or DSA. The aneurysm was either clipped or coiled within 48 hours after bleeding. All patients were kept at the intensive care unit between Day 0 and at least Day 14 post-aSAH. Blood flow velocity (BFV) was routinely measured using TCD on a daily basis, starting with the day of admission. A mean BFV > $120 \mathrm{~cm} / \mathrm{sec}$ was defined as TCD-confirmed vasospasm. ${ }^{11}$ DIND was defined as new neurological deterioration (e.g., aphasia, hemiparesis, confusion, consciousness alteration, and so on) after exclusion of hydrocephalus, rebleeding, seizures, and metabolic disturbances. ${ }^{9}$ Routine CT scans were performed in every patient within 1 day after aneurysm treatment to rule out treatment-induced ischemia and on Day 10 after bleeding. In patients who underwent aneurysm clipping, CTA was additionally obtained with the first postoperative native CT scan (i.e., without application of contrast media). DCI was defined as hypodensity on CT scan, which was not visible on the posttreatment CT scan and after excluding other causes. All patients received intravenous nimodipine for 14 days. Normovolemia was maintained in patients without TCD-vasospasm. In any patient with TCD-vasospasm, noradrenaline was used to induce arterial hypertension (target systolic blood pressure 160-180 mm Hg). Some patients with symptomatic vasospasm and CTP-proven perfusion deficits in the corresponding vessel territory, despite maximal conservative antivasospastic treatment, underwent either intraarterial application of nimodipine, balloon dilation, or a combination of both.

\section{Use of the CTP Study}

\section{The CTP Algorithm}

Investigation with CTP was routinely performed in all patients in the acute phase (Days 3-5) after aSAH. In cases of rapidly progressive TCD-vasospasm (BFV increase $>50 \mathrm{~cm} / \mathrm{sec}$ within 24 hours), CTP was repeated. Additionally, patients who were comatose and/or sedated were routinely investigated with CTP on Day 7 after aSAH. Thus, noncomatose and/or nonsedated patients without TCD-vasospasm were imaged once, those with TCD-vasospasm at least twice; comatose and/or sedated patients without TCD-vasospasm were imaged twice, and those with TCD-vasospasm at least 3 times.

\section{Imaging Protocol}

All images were obtained on a 128 -slice multidetector CT scanner (Siemens Definition AS+; Siemens Healthcare Sector). The following parameters were used for nonenhanced spiral neuro mode scan, covering skull base and the cerebrum with a caudal-cranial range of $12 \mathrm{~cm}: 120$ $\mathrm{kV}, 450 \mathrm{mAs}$, rotation time 1.0 second, maximum pitch 0.8 , slice collimation $50 \times 0.6 \mathrm{~mm}$, kernel H31f, 512 matrix. Routine axial and coronal maximum-projection reconstructions were made, with a 5-mm slice thickness and a 1-mm interslice distance.

The CTP data were acquired using a periodic spiral approach (adaptive 4-dimensional spiral mode) of 30 pe- 
riodic spiral mode scans of the brain with cranial-caudal coverage of $96 \mathrm{~mm}$ in z-axis (1.5-second sampling time per volume). The CTP scan $(80 \mathrm{kV}, 200 \mathrm{mAs}$, rotation time 0.3 seconds, maximum pitch 0.5 , collimation $2 \times 64$ $\times 0.6 \mathrm{~mm}$ ) was started 4 seconds after the injection of a short contrast bolus (Imeron 400; Bracco Imaging) into a cubital vein. The contrast volume was $36 \mathrm{ml}$ at a flow rate of $6 \mathrm{ml} / \mathrm{sec}$, followed by a $30 \mathrm{ml}$ saline chaser at $6 \mathrm{ml} / \mathrm{sec}$.

For CTA $(120 \mathrm{kV}, 120 \mathrm{mAs}$, rotation time 0.3 seconds, pitch 0.6 , collimation $2 \times 64 \times 0.6 \mathrm{~mm}$ ), another $60 \mathrm{ml}$ of contrast agent was injected with a biphasic protocol of 45 $\mathrm{ml}$ at $6 \mathrm{ml} / \mathrm{sec}$ and $15 \mathrm{ml}$ at $3 \mathrm{ml} / \mathrm{sec}$ followed by a $30-$ $\mathrm{ml}$ saline chaser at $3 \mathrm{ml} / \mathrm{sec}$. Routine reconstructions of CTP data were made with a slice width of $5 \mathrm{~mm}$ every 3 mm (kernel H20f, 512 matrix). CTP data were analyzed on a multimodality workstation (Siemens syngo 2010B) equipped with a commercially available software package (Volume Perfusion CT Neuro; Siemens), which calculates quantitative color-coded 3D maps of various cerebral perfusion parameters using a delay-invariant deconvolution method. The peak arterial scan was assigned by region of interest (ROI) analysis in the proximal $\mathrm{A}_{2}$ segment. In addition, CTA data were reconstructed with a slice thickness of $0.75 \mathrm{~mm}$ every $0.4 \mathrm{~mm}$. Axial and coronal reconstructions of maximum-intensity projections (MIP), with a 10$\mathrm{mm}$ slice thickness and a 3-mm interslice distance, were subsequently made. ${ }^{6}$

\section{Data Analysis}

Two experienced neuroradiologists (P.S. and K.D.), who were blinded to all clinical data and the clinical course, assessed the CTP and CTA images. 3D perfusion parameter maps were used to show the distribution of hypoperfusion. Readers were asked to assess the presence of a focal hypoperfused area (defined as a visual abnormality) on any of the parameter maps consisting of cerebral blood flow $(\mathrm{CBF})$, cerebral blood volume (CBV), mean transit time (MTT), time to start (TTS), time to peak (TTP), and time to drain (TTD). If hypoperfusion was present, readers had to assign it to one of the vascular territories. Perfusion abnormalities that persisted on the basis of a primary hemorrhagic event, previous ischemia, or surgical intervention were excluded as acute perfusion deficits. Consensus judgment was determined after reviewing the images independently.

Axial and coronal CTA reconstructions were used to identify vasospasm or occlusion in the following vessels: proximal anterior cerebral artery $\left(\mathrm{A}_{1}\right)$, middle cerebral artery $\left(\mathrm{M}_{1} / \mathrm{M}_{2}\right)$, and the posterior cerebral artery $\left(\mathrm{P}_{1}\right)$. In cases with additional DSA, DSA was also used to spot vessel abnormalities. By definition, cerebral vasospasm is a focal narrowing of vessel diameter compared with the same segment in the initial CTA at the time of SAH. A vessel narrowing $<50 \%$ was considered as mild, $50 \%-$ $75 \%$ as moderate, and $>75 \%$ as high-grade angiographic vasospasm.

In all patients, CTP parameter maps were used to make treatment decisions. Patients with symptoms of vasospasm, increased BFV in TCD, abnormal CTP data (defined as highly visual abnormality on the parameter maps in more than one-third of 1 or more vascular territories), and ar- terial vasospasm on single-phase CTA axial and coronal MIP reconstructions underwent DSA, with the possibility to perform endovascular treatment. ${ }^{6}$ The perfusion deficits were divided into 2 main groups of territorial and nonterritorial. Territorial perfusion deficits were diagnosed if the perfusion deficit was confined to a vessel territory (Fig. 1A). Nonterritorial perfusion deficits were diffuse deficits, including multiple vessel territories or the watershed area between 2 vessel territories (Fig. 1B).

\section{Statistical Analysis}

Fisher's exact test was used to evaluate the sensitivity and specificity of perfusion deficits on CTP for the identification of patients at risk for developing DIND and tissue at risk for DCI, respectively. We used a paired t-test to assess the correlation between perfusion deficit on the CTP and DIND/DCI.

\section{Results}

A total of 43 patients were included in the study. The mean age of patients was 52.0 (29-82) years. Thirty patients were women and 13 were men. In all patients, aSAH was diagnosed within 24 hours after symptom onset, which was considered the moment of aneurysm rupture. Patients with history of minor leak and patients with ill-defined symptom onset were excluded. Multiple aneurysms were identified in 14 patients. By dichotomizing the Hunt and Hess grade into good (Grades I-III) and poor (Grades IV and V), we found a good Hunt and Hess grade in 23 of the 43 patients (53\%). Thirty-nine of the 43 patients (91\%) had a Fisher Grade 3 or 4 on the initial CT scan. The ruptured aneurysm was clipped in 25 patients and coiled in 18 patients. The posttreatment CT scan showed partial cerebellar infarction in 2 patients with coiled basilar artery aneurysm and infarction of the recurrent artery of Heubner in 1 patient with clipped anterior communicating artery aneurysm. The CT scan ruled out spatula-induced hypodensity/ contusion in the frontal and temporal lobes in all clipped aneurysms. There was neither vasospasm in the postsurgery CTA, which was performed with the native CT, nor in the final angiographic series after completion of coiling. A summary of patient characteristics is given in Table 1.

\section{Incidence of DIND and DCl in the Study Population}

Delayed ischemic neurological deficits occurred in 13 of the 43 patients $(30 \%)$ within 2 weeks after aSAH, of whom 7 patients $(54 \%)$ had a transient and 6 patients (46\%) a permanent DIND. Vasospasm-associated DCI developed in 11 of the 43 patients (26\%).

\section{Early CTP (on Days 3-5) for Identification of Patients at Risk for DIND and of Tissue at Risk for DCI}

In 39 of 43 patients (91\%), early CTP was done on Day 3 after aSAH, in 1 patient (2\%) on Day 4, and in 3 patients on Day 5 (7\%); a delay of imaging beyond Day 3 occurred because of a nonfunctioning CT scanner. Perfusion deficits in CTP on Days 3-5 were seen in 14 of the 43 patients (33\%), of whom 10 (72\%) developed DIND (in 4 of these 10 patients, DIND occurred on Days 3-5 and in 6 patients 

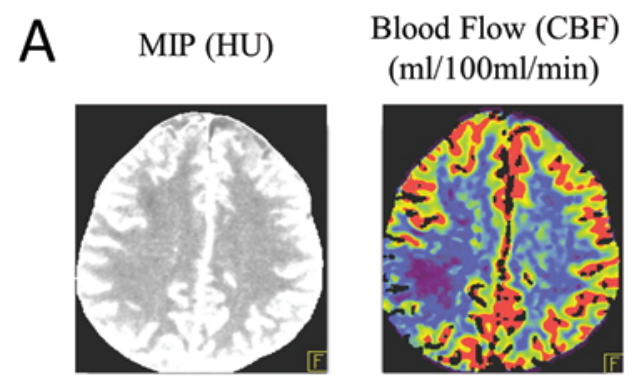
Blood Volume (CBV) $(\mathrm{ml} / 100 \mathrm{~m})$

Time to start

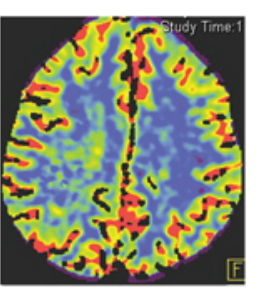

(TTS) (s)

Time to peak

(TTP) (s)

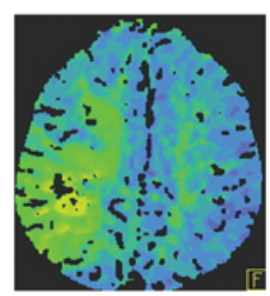

Time to drain

(TTD) (s)
Mean transit time (MTT) (s)
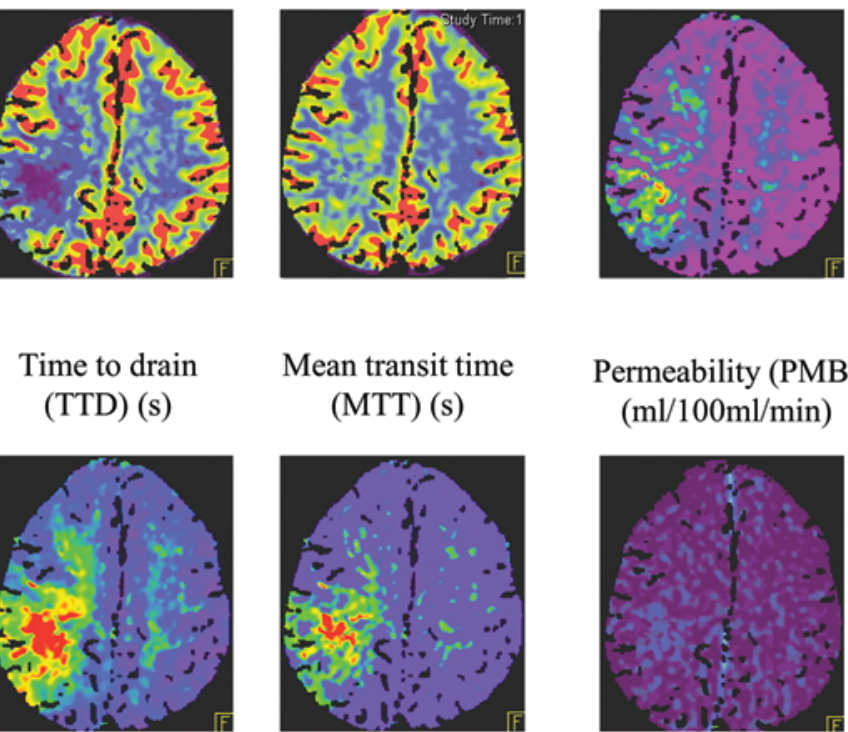

\section{Permeability (PMB)} $(\mathrm{ml} / 100 \mathrm{ml} / \mathrm{min})$

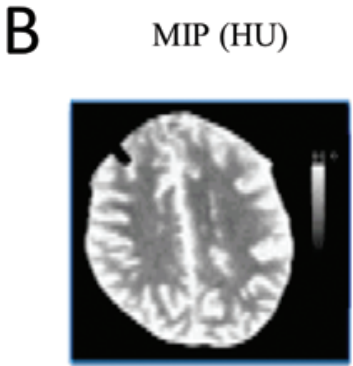

Time to peak

(TTP) (s)

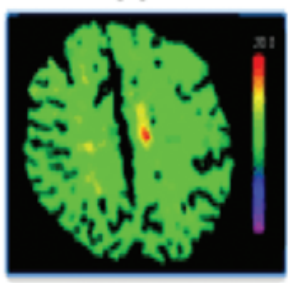

Blood Flow (CBF) Blood Volume (CBV) $(\mathrm{ml} / 100 \mathrm{ml} / \mathrm{min})$

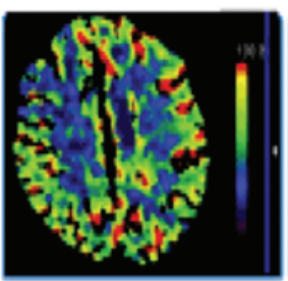

Time to drain

(TTD) (s)

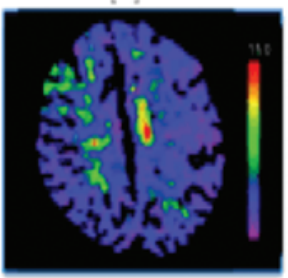

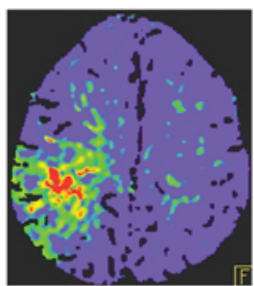

$(\mathrm{ml} / 100 \mathrm{~m})$

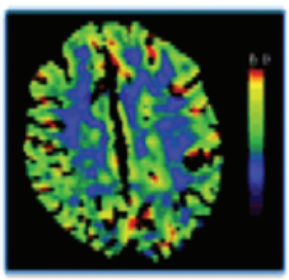

Mean transit time (MTT) (s)

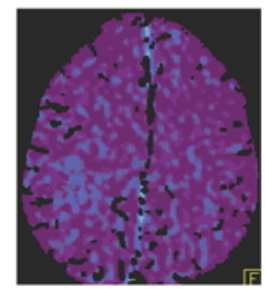

FIG. 1. Whole-brain CTP with perfusion deficits in the middle cerebral artery territory on the right side (A) and diffuse unspecific deficits (B). Figure is available in color online only.

on Days 5-14 after aSAH). Seven of the 10 patients (70\%) with early perfusion deficits and DIND had territorial deficits and 3 patients $(30 \%)$ had nonterritorial deficits.

The remaining 4 patients $(28 \%)$ with early perfusion deficits (but without DIND) had nonterritorial perfusion deficits. Three patients (21\%) developed DCI without preceding DIND, and 1 patient $(7 \%)$ had neither DIND nor DCI. Three patients without early perfusion deficits in CTP later developed DIND; in these patients, a second CTP after Day 5 documented territorial perfusion deficits.

Delayed cerebral infarction occurred in 9 of the 14 patients $(64 \%)$ with early perfusion deficits, of whom 6 patients $(67 \%)$ had preceding DIND. In the remaining 3 pa- tients (33\%), DCI occurred without DIND. Two additional patients developed DCI, but did not demonstrate early perfusion deficits on CTP nor did they have preceding DIND.

The sensitivity and specificity of perfusion deficits on early CTP for the identification of patients at risk for developing DIND was $77 \%$ (95\% CI 46\%-95\%) and $87 \%$ (95\% CI 69\%-96\%), respectively, with a statistically significant correlation between early perfusion deficits on the CTP and the occurrence of DIND after aSAH (Pearson $r$ $=0.62,95 \%$ CI $40 \%-79 \%, p<0.0001)$. The positive predictive value (PPV) was $71 \%(95 \%$ CI $42 \%-92 \%)$ and the negative predictive value (NPV) was 90\% (95\% CI 73\%98\%) (Fig. 2A). 
TABLE 1. Clinical and demographic characteristics of 43 patients with aSAH*

\begin{tabular}{|c|c|}
\hline Characteristic & Value \\
\hline Patients & $43(100)$ \\
\hline Mean age in yrs, range & $52.04,29-82$ \\
\hline \multicolumn{2}{|l|}{ Sex } \\
\hline M & $13(30)$ \\
\hline $\mathrm{F}$ & $30(70)$ \\
\hline \multicolumn{2}{|l|}{ Hunt \& Hess grade } \\
\hline I & 9 \\
\hline ॥ & 8 \\
\hline III & 6 \\
\hline IV & 6 \\
\hline V & 14 \\
\hline \multicolumn{2}{|l|}{ Fisher grade } \\
\hline 1 & 0 \\
\hline 2 & 4 \\
\hline 3 & 25 \\
\hline 4 & 14 \\
\hline \multicolumn{2}{|l|}{ No. of aneurysms } \\
\hline Singular & $29(32)$ \\
\hline Multiple & $14(68)$ \\
\hline \multicolumn{2}{|l|}{ Aneurysm site, $n$} \\
\hline MCA & 15 \\
\hline $\mathrm{ACoA}$ & 11 \\
\hline PCoA & 4 \\
\hline BA & 8 \\
\hline PICA & 1 \\
\hline VA & 1 \\
\hline Pericallosal & 1 \\
\hline ICA & 2 \\
\hline \multicolumn{2}{|l|}{ Aneurysm treatment } \\
\hline Coiling & $18(41)$ \\
\hline Clipping & $25(59)$ \\
\hline
\end{tabular}

$\mathrm{ACOA}=$ anterior communicating artery; $\mathrm{BA}=$ basilar artery; $\mathrm{ICA}=$ internal carotid artery; $\mathrm{MCA}=$ middle cerebral artery; $\mathrm{PCOA}=$ posterior communicating artery; PICA = posterior inferior cerebellar artery; $\mathrm{VA}=$ vertebral artery.

* Values are expressed as the number (\%).

Early CTP identified tissue at risk for developing vasospasm-associated DCI with a sensitivity of $82 \%(95 \%$ CI $48 \%-98 \%)$ and a specificity of $84 \%(95 \%$ CI $67 \%-95 \%)$. The PPV was 64\% (95\% CI 35\%-87\%) and the NPV was 93\% (95\% CI 77\%-99\%). Early perfusion deficits on CTP and the occurrence of vasospasm-associated DCI correlated significantly (Pearson $\mathrm{r}=0.62,95 \%$ CI $39 \%-77 \%$, $\mathrm{p}$ $=0.0001)($ Fig. $2 \mathrm{~B})$.

\section{Use of CTP in Rapidly Progressive TCD-Vasospasm (daily increase in mean BFV $>50 \mathrm{~cm} / \mathrm{sec}$ )}

A rapid increase of TCD-measured mean BFV of $>50$ $\mathrm{cm} / \mathrm{sec}$ within 24 hours was detected in 8 of the 43 patients (19\%). Despite the steep BFV increase, no perfusion deficit could be detected in 5 of the 8 patients $(62.5 \%)$.
None of these 5 patients developed DCI. However, 2 of the 5 patients without perfusion deficit later developed DIND. In the remaining 3 patients $(37.5 \%)$, nonterritorial perfusion deficits were seen on CTP, of whom 2 patients (67\%) developed DCI without preceding DIND and 1 patient (33\%) had DIND without DCI. The sensitivity and specificity of CTP in rapidly progressive TCD-vasospasm for identification of patients at risk for DIND were 33\% (95\% CI 8\%-90\%) and 60\% (95\% CI 14\%-94\%), respectively, and the PPV and NPV were 33\% (95\% CI 9\%-90\%) and $60 \%$ (95\% CI 14\%-94\%), respectively. The sensitivity and specificity of CTP in rapidly progressive TCD-vasospasm for identification of tissue at risk were $100 \%(95 \%$ CI $15 \%-100 \%)$ and $83 \%$ (95\% CI 35\%-99\%), respectively, and the PPV and NPV were 67\% (95\% CI 9\%-99\%) and $100 \%$ (95\% CI 47\%-100\%), respectively.

\section{Use of CTP on Day 7 in Patients Who Were Comatose and/ or Sedated}

Seven of the 43 patients (16\%) who were comatose and/ or sedated on Day 7 after the initial bleeding received an additional CTP on Day 7. Four of these 7 patients (57\%) had perfusion deficits on the CTP on Day 7, of whom 3 patients had nonterritorial and 1 patient had a territorial perfusion deficit. All 4 patients with perfusion deficits developed DCI. None of the 3 patients without perfusion deficits developed DCI.

\section{Discussion}

CT perfusion is a well-established tool in the imaging of ischemic stroke. Thus, it is not surprising that CTP was also used in the diagnostic workup after aSAH, with the aim to detect impending vasospastic infarction during the so-called vasospasm period between Days 5 and 14 after the initial bleeding. ${ }^{16,24,36}$

\section{Earlier Studies}

Wintermark et al. performed a retrospective analysis of 27 patients with aSAH who were suspected to develop angiographic vasospasm and therefore underwent CTP/ CTA, TCD, and DSA between Days 6 and 8 after the initial bleeding. They found a significant correlation of CTP/CTA findings with those of DSA. The most accurate CTP parameter (based on ROI analysis) in this study for the detection of angiographic vasospasm was MTT, with a cutoff at 6.4 seconds and a very high NPV of $98.7 \% .^{36}$ Sanelli et al. performed a retrospective analysis of a single ROI-based CTP in 75 patients between Day 0 and Day 3 after aSAH and found a statistically significant reduction of CBF and prolongation of MTT on early CTP in patients who develop angiographic vasospasm later. ${ }^{28}$ In a second retrospective study in 96 patients with aSAH, Sanelli et al. performed ROI-based CTP between Days 6 and 8 after aSAH and found a sensitivity of $78 \%$ and a specificity of $66 \%$ of CTP for the development of permanent neurological deficits and a sensitivity of $88 \%$ and a specificity of $59 \%$ for the development of DCI. ${ }^{29}$

In a retrospective ROI-based CTP study by Kunze et al., 53 patients with aSAH were examined on Day 3 or 4, Day 6 or 7, Days 9 and 10, and additionally at any other 

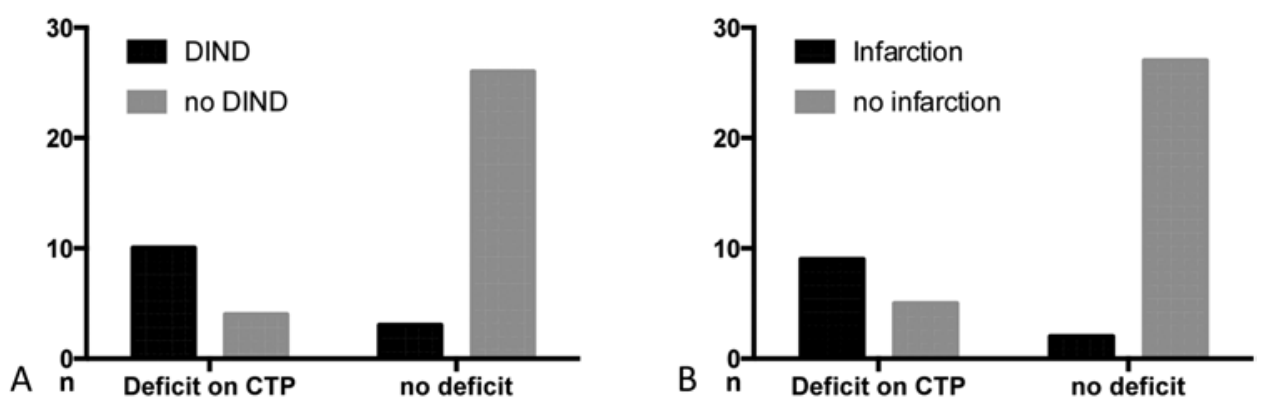

FIG. 2. Correlation of early deficit on CTP with the incidence of DIND (A) and infarction (B).

time point when cerebral vasospasm was suspected..$^{18}$ The CTP parameter with the highest sensitivity (93\%) but a low specificity (27\%) for the detection of angiographic vasospasm was TTP. ${ }^{18}$ With at least 3 CTP investigations per patient, the exposure to radiation was higher than in most other studies and in the present study. Killeen et al. performed ROI-based CTP in 97 consecutive aSAH patients between Days 6 and 8 and correlated in a retrospective analysis the CTP data with cerebral infarction and/or permanent neurological deficit. The sensitivity and specificity for the detection of infarction and/or permanent neurological deficit were $84 \%$ and $73 \%$, respectively. ${ }^{16}$ Etminan and colleagues performed a retrospective study in 51 patients; they showed that an MTT increase within 12 hours after bleeding but before therapy is related to poor outcome?

In a prospective study, Hickmann et al. included 38 patients with aSAH. CTP and TCD measurements were performed on Days 3, 7, and 10 after aneurysm rupture. Hickmann and colleagues performed a semiquantitative and visual CTP analysis using 6 predefined ROIs. They concluded that TTP was the most sensitive and most specific predictor of clinically relevant vasospasm, defined as cerebral infarction and/or DIND (sensitivity of $90 \%$ and specificity of $61.1 \%) .{ }^{13}$ In a prospective study by Dankbaar et al., ROI-based CTP was performed at the time point of clinical deterioration in 42 patients with aSAH. The primary end point was the development of new focal neurological deficit or decreased Glasgow Coma Scale score of $>2$ points, lasting $>2$ hours. A vasospasm-induced new neurological deficit occurred in 25 patients. CTP had a sensitivity of $84 \%$ and a specificity of $79 \%$ for the diagnosis of new focal neurological deficit due to vasospasm at the time point of clinical deterioration. ${ }^{4}$

All of these studies have significant limitations. One of them is validating parameter. In the studies by Wintermark et al., Sanelli et al., and Kunze et al., the role of CTP for cerebral vasospasm detection was defined by comparing CTP with DSA. However, only a minority of patients with angiographic vasospasm develop symptomatic vasospasm and possibly need initiation or intensification of the vasospastic therapy. Killeen et al. and, in part, Hickmann et al. compared CTP findings with DCI or permanent neurological deficit. However, the potential benefit of CTP is identification of patients and tissue at risk prior to infarction and fixed neurological deficits. Etminan et al. used outcome as a parameter for defining the value of CTP performed within 12 hours after aSAH and before therapy. Outcome after aSAH is not only related to vasospasm and DCI, but also parameters that are not closely linked to brain perfusion, such as age, hydrocephalus, and modality of aneurysm therapy. Thus, outcome might not be the ideal validating parameter.

A second limitation is time point and repetition of CTP investigation. In the current literature, an accepted time point for CTP does not exist. In some series, CTP was performed only in cases of clinical deterioration; in other series, up to 3 routine CTP scans were obtained. ${ }^{4,13,16,18,28,36}$ However, CTP at the moment of neurological decline might be too late for initiation of a successful antivasospastic therapy, whereas 3 routine CTPs might unnecessarily expose patients to radiation.

The ROIs represent a third limitation. Prediction of vascular territories in which hypoperfusion might occur is difficult. Therefore, preselection of ROIs carries the risk that areas of brain hypoperfusion remain undetected. Another limitation is study design; the vast majority of the published studies were retrospective analyses.

\section{Rationale for the Present Study}

The rationale for study initiation was to attempt to overcome some of the aforementioned limitations of other studies. We used an imaging algorithm to 1) identify patients and tissue at risk before occurrence of fixed neurological deficits and DCI, 2) reduce the number of repeated scans to a minimum, and 3) obtain CTP data from defined and therefore comparable time points after aSAH. We planned to perform the first CTP on Day 3 for 2 reasons. First, TCD studies indicated Days 4-14 to be the vasospasm period. Second, studies by Sanelli et al. and Etminan et al. have shown that patients who later develop angiographic vasospasm already had a CBF reduction and an MTT prolongation on a Day 0 to Day 3 CTP. In surgical cases, spatula pressure could induce prolonged frontal and temporal hypoperfusion; therefore, we refrained from CTP on Days 0-2. We were able to perform CTP on Day 3 in the vast majority of included patients, but CTP had to be postponed in 1 patient to Day 4 and in 3 patients to Day 5 due to a nonfunctioning CT scanner, which might represent a certain limitation of the study. However, according to the clinical and TCD criteria, none of these patients had vasospasm. CTP was repeated if 1) TCD suggested development of clinically relevant vasospasm using the criterion of a BFV increase $>50 \mathrm{~cm} / \mathrm{sec}$ within 24 hours, or 2) intubation and sedation did not allow clinical monitoring. This algorithm was evaluated in a prospective study using whole-brain instead of ROI-based CTP. 


\section{Can CTP on Day 3 Predict DIND and DCl?}

In the present study, $31 \%$ of all patients developed DIND, which is in line with the percentage previously described in the literature. ${ }^{27,33}$ The most important result of the study was that whole-brain CTP routinely performed on Day 3 has a high sensitivity and specificity for the identification of patients at risk for the development of DIND, allowing early initiation or intensification of antivasospastic therapy. The sensitivity was $77 \%$, which is comparable with the reported sensitivity of CTP in the vasospasm phase and only slightly lower compared with the sensitivity of CTP performed at the time of clinical deterioration, which could be too late to avoid permanent neurological deficits or infarction. The specificity of early whole-brain CTP was $87 \%$, which was even higher than in other studies. Our results clearly indicate that whole-brain CTP scanning on Day 3 after aSAH has sufficient diagnostic accuracy with the lowest possible radiation exposure; it provides an early diagnosis, allowing for initiation of appropriate treatment.

\section{Can TCD, as a Pretest, Reduce the Number of Additional CTP Investigations?}

Transcranial Doppler sonography is an established monitoring tool for the identification of cerebral vasospasm after aSAH. With the aim to reduce the number of repeated CTP scans, we tried to use TCD for identification of patients at risk. Repeat CTP was only performed in patients with a BFV increase $>50 \mathrm{~cm} / \mathrm{sec}$ within 24 hours. This was based on the study by Rätsep et al., who found a correlation of cerebral hemodynamic impairment with severe vasospasm and with a rapid increase in TCD-BFV. ${ }^{26}$ We showed that an increase in TCD-BFV usually lags 1 or 2 days behind the identification of perfusion deficits shown by CTP. Accordingly, we found no statistically significant correlation between TCD-BFV increase and perfusion deficits on CTP. Thus, we can conclude that the parameter of TCD-BFV increase alone is not suitable for the detection of tissue at risk.

Concerning the detection of tissue at risk, whole-brain CTP should be an essential part of the diagnostic workup in addition to daily clinical assessment and daily BFV measurement by TCD. These findings are supported by the study by Pham et al., which evaluated the diagnostic accuracy of CTP and TCD for the prediction of DCI between Days 3 and 14 after aSAH. They performed qualitative visual analysis of the CTP maps and quantitative analysis based on predefined ROIs and found a good prediction of DCI by CTP at a median of 3 days before infarction manifestation. In line with our findings, TCD measurements failed to predict the occurrence of DCI. ${ }^{24} \mathrm{In}$ contrast, in a retrospective study of 45 patients with aSAH, Toi et al. reported that TCD in the early stage of aSAH can predict the future occurrence of symptomatic vasospasm. In their study, the highest sensitivity (71.4\%) and specificity $(68.1 \%)$ were seen on Day 3, with a BFV threshold of $72.5 \mathrm{~cm} / \mathrm{sec}^{31}$

\section{Whole-Brain CTP on Day 7 After aSAH in Patients Who Were Comatose and/or Sedated}

The identification of impending DIND or DCI in pa- tients with high-grade aSAH is difficult due to a decreased possibility of neurological assessment. Therefore, we repeated CTP on Day 7 after aSAH in patients who were comatose and/or sedated. Perfusion deficits were seen in 4 of the 7 patients who were comatose and/or sedated. Interestingly, the initial CTP had already demonstrated perfusion deficits in these patients, of whom all developed DCI. This suggests that the information gain by repeated CTP on Day 7 in patients who are comatose and/or sedated is low and too late for initiation of appropriate antivasospastic therapy. However, the number of patients is far too low to draw any final conclusion concerning the diagnostic value of CTP on Day 7 after aSAH in patients who are comatose and/or sedated.

\section{Does CTP Improve Outcome?}

The overall aim of using TCD and DSA for vasospasm identification, and of using CTP for identification of tissue at risk for DCI, was to improve outcome. We showed that early CTP has a high PPV and NPV for DIND and DCI. However, the study design did not allow us to determine if that high PPV translates into better outcome. All patients with proven territorial hypoperfusion in CTP and artery narrowing in CTA underwent further endovascular therapy and/or induced hypertension. However, the treatment was not standardized for purposes of the study, and treatment failure, which is frequent in patients with severe vasospasm despite timely recognition of tissue at risk by CTP, was not defined. We strongly believe that a larger study, which perhaps could not be conducted at a single center, would be necessary to address the issue of outcome improvement by CTP.

The study also did not allow us to answer the question of whether CTP is superior to routine angiography during the vasospasm period or to measurement of $\mathrm{ptiO}_{2}$ (brain tissue oxygen partial pressure), because no comparison between the tools was performed. However, the study allowed us to gain information on the value of CTP compared with clinical examination and TCD. We showed that 1) CTP on Day 3 (i.e., before the majority of patients become clinically symptomatic) has a high sensitivity and specificity for the identification of patients at risk for the development of DIND, and 2) the increase in TCD-BFV usually lags 1 or 2 days behind the identification of perfusion deficits by CTP. These findings suggest that early CTP might be a better tool than clinical examination and TCD for timely identification of patients at risk.

\section{Strengths and Limitations of the Study}

Strengths of this study include its prospective nature, the use of a rigid CTP algorithm, the focus on an increase in sensitivity as well as an avoidance of unnecessary radiation exposure, and the use of whole-brain CTP in patients with aSAH for the first time. We had to delay early CTP in $9 \%$ of patients to Days 4 and 5 due to logistic reasons, which might represent a limitation. However, none of the patients had clinical signs of vasospasm or BFV $>120 \mathrm{~cm} /$ sec. The number of included patients is still too small to consistently answer distinct questions in subgroup analyses. Furthermore, the results of the present study, as well as those of previous studies concerning sensitivity and speci- 
ficity, are hampered by the fact that the CTP findings led to initiation of therapies with varying efficacy to reverse the detected perfusion deficits.

\section{Conclusions}

Whole-brain CTP is a rapid imaging technique that allows early identification of patients at risk for developing DIND and tissue at risk for vasospasm-induced infarction within days after aSAH. On the basis of this study's findings, a routine use of whole-brain CTP in the acute phase of aSAH seems to be useful.

\section{References}

1. Aralasmak A, Akyuz M, Ozkaynak C, Sindel T, Tuncer R: CT angiography and perfusion imaging in patients with subarachnoid hemorrhage: correlation of vasospasm to perfusion abnormality. Neuroradiology 51:85-93, 2009

2. Bederson JB, Connolly ES Jr, Batjer HH, Dacey RG, Dion JE, Diringer MN, et al: Guidelines for the management of aneurysmal subarachnoid hemorrhage: a statement for healthcare professionals from a special writing group of the Stroke Council, American Heart Association. Stroke 40:994-1025, 2009

3. Chaudhary SR, Ko N, Dillon WP, Yu MB, Liu S, Criqui GI, et al: Prospective evaluation of multidetector-row CT angiography for the diagnosis of vasospasm following subarachnoid hemorrhage: a comparison with digital subtraction angiography. Cerebrovasc Dis 25:144-150, 2008

4. Dankbaar JW, de Rooij NK, Velthuis BK, Frijns CJM, Rinkel GJE, van der Schaaf IC: Diagnosing delayed cerebral ischemia with different CT modalities in patients with subarachnoid hemorrhage with clinical deterioration. Stroke 40:3493-3498, 2009

5. Darwish RS, Ahn E, Amiridze NS: Role of transcranial Doppler in optimizing treatment of cerebral vasospasm in subarachnoid hemorrhage. J Intensive Care Med 23:263-267, 2008

6. Dolatowski K, Malinova V, Frölich AM, Schramm R, Haberland U, Klotz E, et al: Volume perfusion CT (VPCT) for the differential diagnosis of patients with suspected cerebral vasospasm: qualitative and quantitative analysis of 3D parameter maps. Eur J Radiol 83:1881-1889, 2014

7. Etminan N, Beseoglu K, Heiroth HJ, Turowski B, Steiger HJ, Hänggi D: Early perfusion computerized tomography imaging as a radiographic surrogate for delayed cerebral ischemia and functional outcome after subarachnoid hemorrhage. Stroke 44:1260-1266, 2013

8. Fontanella M, Valfrè W, Benech F, Carlino C, Garbossa D, Ferrio M, et al: Vasospasm after SAH due to aneurysm rupture of the anterior circle of Willis: value of TCD monitoring. Neurol Res 30:256-261, 2008

9. Frontera JA, Fernandez A, Schmidt JM, Claassen J, Wartenberg KE, Badjatia N, et al: Defining vasospasm after subarachnoid hemorrhage: what is the most clinically relevant definition? Stroke 40:1963-1968, 2009

10. Frölich AM, Psychogios MN, Klotz E, Schramm R, Knauth M, Schramm P: Angiographic reconstructions from wholebrain perfusion CT for the detection of large vessel occlusion in acute stroke. Stroke 43:97-102, 2012

11. Gonzalez NR, Boscardin WJ, Glenn T, Vinuela F, Martin NA: Vasospasm probability index: a combination of transcranial Doppler velocities, cerebral blood flow, and clinical risk factors to predict cerebral vasospasm after aneurysmal subarachnoid hemorrhage. J Neurosurg 107:1101-1112, 2007

12. Greenberg ED, Gobin YP, Riina H, Johnson CE, Tsiouris AJ, Comunale J, et al: Role of CT perfusion imaging in the diag- nosis and treatment of vasospasm. Imaging Med 3:287-297, 2011

13. Hickmann AK, Langner S, Kirsch M, Baldauf J, Müller C, Khaw A, et al: The value of perfusion computed tomography in predicting clinically relevant vasospasm in patients with aneurysmal subarachnoid hemorrhage. Neurosurg Rev 36:267-278, 2013

14. Honda M, Sase S, Yokota K, Ichibayashi R, Yoshihara K, Sakata Y, et al: Early cerebral circulatory disturbance in patients suffering subarachnoid hemorrhage prior to the delayed cerebral vasospasm stage: xenon computed tomography and perfusion computed tomography study. Neurol Med Chir (Tokyo) 52:488-494, 2012

15. Kamp MA, Heiroth HJ, Beseoglu K, Turowski B, Steiger HJ, Hänggi D: Early CT perfusion measurement after aneurysmal subarachnoid hemorrhage: a screening method to predict outcome? Acta Neurochir Suppl 114:329-332, 2012

16. Killeen RP, Gupta A, Delaney H, Johnson CE, Tsiouris AJ, Comunale J, et al: Appropriate use of CT perfusion following aneurysmal subarachnoid hemorrhage: a Bayesian analysis approach. AJNR Am J Neuroradiol 35:459-465, 2014

17. Killeen RP, Mushlin AI, Johnson CE, Comunale JP, Tsiouris AJ, Delaney H, et al: Comparison of CT perfusion and digital subtraction angiography in the evaluation of delayed cerebral ischemia. Acad Radiol 18:1094-1100, 2011

18. Kunze E, Pham M, Raslan F, Stetter C, Lee JY, Solymosi L, et al: Value of perfusion CT, transcranial Doppler sonography, and neurological examination to detect delayed vasospasm after aneurysmal subarachnoid hemorrhage. Radiol Res Pract 2012:231206, 2012

19. Lagares A, Cicuendez M, Ramos A, Salvador E, Alén JF, Kaen A, et al: Acute perfusion changes after spontaneous SAH: a perfusion CT study. Acta Neurochir (Wien) 154:405-412, 2012

20. Macdonald RL: Delayed neurological deterioration after subarachnoid haemorrhage. Nat Rev Neurol 10:44-58, 2014

21. Mills JN, Mehta V, Russin J, Amar AP, Rajamohan A, Mack WJ: Advanced imaging modalities in the detection of cerebral vasospasm. Neurol Res Int 2013:415960, 2013

22. Miranda P, Lagares A, Alen J, Perez-Nuñez A, Arrese I, Lobato RD: Early transcranial Doppler after subarachnoid hemorrhage: clinical and radiological correlations. Surg Neurol 65:247-252, 2006

23. Moftakhar R, Rowley HA, Turk A, Niemann DB, Kienitz BA, Van Gomple J, et al: Utility of computed tomography perfusion in detection of cerebral vasospasm in patients with subarachnoid hemorrhage. Neurosurg Focus 21(3):E6, 2006

24. Pham M, Johnson A, Bartsch AJ, Lindner C, Müllges W, Roosen K, et al: CT perfusion predicts secondary cerebral infarction after aneurysmal subarachnoid hemorrhage. Neurology 69:762-765, 2007

25. Psychogios MN, Schramm P, Frölich AM, Kallenberg K, Wasser K, Reinhardt L, et al: Alberta Stroke Program Early CT Scale evaluation of multimodal computed tomography in predicting clinical outcomes of stroke patients treated with aspiration thrombectomy. Stroke 44:2188-2193, 2013

26. Rätsep T, Asser T: Cerebral hemodynamic impairment after aneurysmal subarachnoid hemorrhage as evaluated using transcranial Doppler ultrasonography: relationship to delayed cerebral ischemia and clinical outcome. J Neurosurg 95:393-401, 2001

27. Rowland MJ, Hadjipavlou G, Kelly M, Westbrook J, Pattinson KT: Delayed cerebral ischaemia after subarachnoid haemorrhage: looking beyond vasospasm. Br J Anaesth 109:315-329, 2012

28. Sanelli PC, Jou A, Gold R, Reichman M, Greenberg E, John $\mathrm{M}$, et al: Using CT perfusion during the early baseline period in aneurysmal subarachnoid hemorrhage to assess for development of vasospasm. Neuroradiology 53:425-434, 2011 
29. Sanelli PC, Anumula N, Johnson CE, Comunale JP, Tsiouris AJ, Riina $\mathrm{H}$, et al: Evaluating $\mathrm{CT}$ perfusion using outcome measures of delayed cerebral ischemia in aneurysmal subarachnoid hemorrhage. AJNR Am J Neuroradiol 34:292298, 2013

30. Simm RF, de Aguiar PH, de Oliveira Lima M, Paiva BL: Transcranial Doppler as a routine in the treatment of vasospasm following subarachnoid hemorrhage (SAH). Acta Neurochir Suppl (Wien) 115:75-76, 2013

31. Toi H, Matsumoto N, Yokosuka K, Matsubara S, Hirano K, Uno M: Prediction of cerebral vasospasm using early stage transcranial Doppler. Neurol Med Chir (Tokyo) 53:396402, 2013

32. Tsivgoulis G, Alexandrov AV, Sloan MA: Advances in transcranial Doppler ultrasonography. Curr Neurol Neurosci Rep 9:46-54, 2009

33. Wachter D, Hans F, Kreitschmann-Andermahr I, Rohde V: Lower incidence of transcranial Doppler and symptomatic vasospasm after aneurysmal subarachnoid hemorrhage and aneurysm clipping in the elderly patient? Neurosurgery 69:261-267, 2011

34. Williams JR: The Declaration of Helsinki and public health. Bull World Health Organ 86:650-652, 2008

35. Wintermark M, Dillon WP, Smith WS, Lau BC, Chaudhary S, Liu S, et al: Visual grading system for vasospasm based on perfusion CT imaging: comparisons with conventional angiography and quantitative perfusion CT. Cerebrovasc Dis 26:163-170, 2008

36. Wintermark M, Ko NU, Smith WS, Liu S, Higashida RT, Dillon WP: Vasospasm after subarachnoid hemorrhage: utility of perfusion CT and CT angiography on diagnosis and management. AJNR Am J Neuroradiol 27:26-34, 2006

37. Xyda A, Haberland U, Klotz E, Bock HC, Jung K, Knauth M, et al: Brain volume perfusion CT performed with 128-detec- tor row CT system in patients with cerebral gliomas: a feasibility study. Eur Radiol 21:1811-1819, 2011

38. Xyda A, Haberland U, Klotz E, Jung K, Bock HC, Schramm $\mathrm{R}$, et al: Diagnostic performance of whole brain volume perfusion CT in intra-axial brain tumors: preoperative classification accuracy and histopathologic correlation. Eur J Radiol 81:4105-4111, 2012

\section{Disclosures}

Prof. Moerer gave lectures on mechanical ventilation and hemodynamic monitoring at regional workshops and industrysponsored sessions within the context of a national congress and received speaker's honoraria from Pulsion Medical, Hill-Rom, and MAQUET Critical Care.

\section{Author Contributions}

Conception and design: Malinova, Moerer, Rohde, Mielke. Acquisition of data: Malinova, Dolatowski, Schramm. Analysis and interpretation of data: Malinova, Dolatowski, Schramm, Rohde, Mielke. Drafting the article: Malinova. Critically revising the article: Schramm, Moerer, Rohde, Mielke. Reviewed submitted version of manuscript: Moerer, Rohde. Approved the final version of the manuscript on behalf of all authors: Malinova. Statistical analysis: Malinova. Administrative/technical/material support: Dolatowski, Schramm. Study supervision: Moerer, Rohde, Mielke.

\section{Correspondence}

Vesna Malinova, Department of Neurosurgery, Georg-AugustUniversity, Robert-Koch-Straße 40, Göttingen 37075, Germany. email: vesna.malinova@gmail.com. 